\title{
Análisis de convergencia para el caso ecuatoriano a nivel cantonal en el período: 2007-2017
}

\section{Analysis of Convergence for the ecuadorian case at the cantonal level in the period: 2007-2017}

José Paúl Tinizhañay Peralta es investigador independiente (jose_tinizhanay@hotmail.com) (https://orcid.org/0000-00027350-693X)

\begin{abstract}
Resumen
El objetivo de este estudio es analizar la existencia de convergencia y determinar su magnitud a nivel cantonal para la economía ecuatoriana durante el período 2007 a 2017. Siguiendo la metodología propuesta por Quah, se estima la matriz de transición de la economía ecuatoriana a nivel cantonal y siguiendo los postulados de Barro y Sala-i-Martin, se identifica el proceso de convergencia sigma y se estima un modelo econométrico utilizando MCO y datos de corte transversal, para determinar la existencia y magnitud de la convergencia beta. Los principales hallazgos indican que Ecuador muestra una mejora en la situación final de movilidad. Además, la tasa de convergencia absoluta asciende al $3.62 \%$ promedio anual en el nivel cantonal, mientras que, al incluir factores de condicionamiento en relación con la infraestructura física de los hogares, el consumo de electricidad y la educación, la velocidad de convergencia asciende al 4,67\% promedio anual, lo que denota áreas clave de intervención, entre otras, para acortar brechas territoriales en Ecuador.
\end{abstract}

\begin{abstract}
The objective of this study is to analyze the existence of convergence and determine its magnitude at the cantonal level for the Ecuadorian economy during the period 2007 to 2017. Following the methodology proposed by Quah, the transition matrix of the Ecuadorian economy at the cantonal level is estimated and following the postulates of Barro and Sala-i-Martin, the sigma convergence process is identified and an econometric model is estimated using OLS and cross-sectional data, to determine the existence and magnitude of the beta convergence. The main findings indicate that Ecuador shows an improvement in the final mobility situation. Furthermore, the absolute convergence rate amounts to $3.62 \%$ annual average at the cantonal level, while, by including conditioning factors in relation to physical infrastructure of households, electricity consumption and education, the speed of convergence amounts to $4.67 \%$ average annual, which denotes key areas of intervention, among others, in order to shorten territorial gaps in Ecuador.
\end{abstract}

Palabras clave I keywords

Matriz de movilidad, Convergencia Sigma, Convergencia Beta, crecimiento económico, desarrollo, corte transversal. Mobility Matrix, Sigma Convergence, Beta Convergence, economic growth, development, cross-sectional data.

Forma sugerida de citar: Tinizhañay Peralta, J. P. (2020). Análisis de convergencia para el caso ecuatoriano a nivel cantonal en el período: 2007-2017. Retos Revista de Ciencias de la Administración y Economía, 10(19), 167-186. https://doi.org/10.17163/ret.n19.2020.10 


\section{Introducción}

El crecimiento económico significa una oportunidad para que un país emerja de la pobreza o mejore sus niveles de vida actuales. Sin embargo, si este crecimiento solo se concentra en ciertas áreas que históricamente han mostrado un alto rendimiento debido a su ubicación geográfica, concentración de entidades públicas, puertos de acceso marítimo y otras ventajas exógenas, entonces existe un problema de polarización de la riqueza y no un verdadero crecimiento económico y desarrollo.

En la última década, Ecuador ha mostrado un período de crecimiento económico y, en consecuencia, el gobierno ha emprendido una serie de medidas con el objetivo, entre muchos otros, de cerrar las brechas entre las zonas ricas y deprimidas del país y así lograr una integración económica visible. Sin embargo, es de gran interés, tanto desde el punto de vista político como académico, encontrar evidencia sobre si este crecimiento y políticas han tenido el efecto deseado o, por el contrario, si se mantiene la concentración de la riqueza en ciertas regiones.

Por lo tanto, la presente investigación empírica tiene como objetivo mostrar la existencia y la magnitud de la convergencia a nivel cantonal entre 2007 y 2017 para el caso ecuatoriano. Para este propósito, se utilizan tres estrategias, la primera es analizar la movilidad de los cantones entre diferentes estados o estratos de acuerdo con su producción anual promedio per cápita para el período 2007 y 2017, a través de la matriz de transición propuesta por Quah. En segundo lugar, para identificar la existencia de convergencia y su magnitud en el tiempo a nivel cantonal, se toman como base los postulados de Barro y Sala-i-Martin sobre convergencia sigma. La tercera estrategia consiste en estimar un modelo econométrico utilizando datos de sección transversal, siguiendo la propuesta de Barro y Sala-i-Martin, para demostrar la convergencia beta condicional. En este sentido, dado el crecimiento económico y la estabilidad política experimentados en Ecuador durante el período de análisis, es posible hipotetizar una movilidad positiva de los cantones, lo que mostraría una mejora de la economía ecuatoriana en su conjunto al final del período analizado. Además, dado el tamaño de Ecuador y su nivel de desarrollo social, es posible pensar que los factores clave en torno a los niveles de ahorro, tecnología, depreciación de capital y movilidad de factores entre los cantones dentro del país pueden ser similares, por lo tanto, es muy probable que se cumplirá la hipótesis de convergencia.

La importancia y originalidad de esta investigación radica en el hecho de incorporar diferentes enfoques para sacar conclusiones sólidas desde el punto de vista académico y científico. Además, la producción literaria realizada hasta el momento sobre este tema es muy escasa para el caso ecuatoriano, limitando el análisis a nivel provincial en períodos anteriores más cortos, que no cubren la sección temporal analizada en este trabajo. En consecuencia, existe una necesidad imperiosa de un análisis de investigación más detallado y exhaustivo que pueda proporcionar un criterio, con rigor científico, para los planificadores y los encargados de formular políticas económicas en el país sobre la situación actual de las disparidades territoriales y el desempeño de la economía a nivel cantonal.

Los principales resultados obtenidos muestran que los cantones ecuatorianos analizados han experimentado movilidad positiva, es decir, al final del período de 
análisis, un porcentaje importante de cantones se encuentran en posiciones relativas más altas en comparación con su situación inicial. Además, la evidencia empírica corrobora la existencia de convergencia sigma y beta condicional para el caso ecuatoriano en el período 2007-2017.

Esta investigación tiene la siguiente estructura: primero, se presenta una sección introductoria, luego, se resume la literatura de referencia principal. Los datos y la metodología empleados se detallan en la segunda sección. Después de eso, se presentan los principales resultados y su discusión. La última sección resume las principales conclusiones y recomendaciones extraídas de los hallazgos presentados.

\section{Revisión de literatura}

Las diferencias en los niveles de crecimiento de las economías de todo el mundo han tratado de ser explicadas a través de varias teorías a lo largo del tiempo. Así, el intercambio de ideas en un entorno académico ha llevado a dos corrientes principales que explican tales diferencias entre países. Primero, la teoría neoclásica del crecimiento económico formulada a partir del trabajo de Solow (1956) argumenta que, dada la existencia de un estado constante, único y estable, accesible independientemente de las condiciones iniciales, se predice una mayor tasa de crecimiento económico para las economías pobres en contraste con las economías ricas, y en consecuencia eventualmente habrá convergencia de las tasas de crecimiento y los niveles de ingreso per cápita.

Por otro lado, las teorías de crecimiento endógeno consideran que la tasa de crecimiento económico depende básicamente de tres factores: capital físico, capital humano y conocimiento o progreso técnico, que son acumulativos y generan externalidades. Al considerar este postulado, los nuevos modelos de crecimiento endógeno reemplazan los postulados neoclásicos de competencia perfecta y rendimientos constantes a escala con competencia imperfecta y rendimientos crecientes. Como resultado, estas nuevas teorías consideran el crecimiento económico como un proceso endógeno del sistema económico (Barro \& Sala-i-Martin, 1991). En esta corriente principal, hay varios trabajos que analizan esta nueva concepción del crecimiento económico como, por ejemplo: Romer (1986), Lucas (1988), Rebelo (1991) y Young (1991), entre otros.

A partir de este último enfoque, Barro y Sala-i-Martin (1991) diseñan una propuesta para abordar el tema de la convergencia, que parte de un modelo neoclásico y predice la existencia de una relación negativa entre el ingreso inicial y la tasa de crecimiento, bajo la premisa de que la diferencia entre economías se debe a sus existencias iniciales de capital físico. Más tarde, Barro y Sala-i-Martin (1992a) formulan una metodología para el estudio de la convergencia que consiste en estimar un modelo de regresión múltiple donde la tasa de crecimiento del PIB per cápita es una función del PIB inicial per cápita y el valor en su estado constante. Sin embargo, esta metodología se ha transformado en un modelo de regresión lineal simple, donde el valor de estado constante del PIB de cada país está contenido en el término de perturbación. Este proceso implica que todos los países se acercan al mismo estado estacionario, que no está correlacionado con su nivel de ingreso per cápita. Sin embargo, esta metodología se ha transformado en un modelo de regresión lineal simple, donde 
el valor de estado constante del PIB de cada país está contenido en el término de perturbación. Este proceso implica que todos los países se acercan al mismo estado estacionario, que no está correlacionado con su nivel de ingreso per cápita.

Además, Barro y Sala-i-Martin (1992b) también definen otro tipo de convergencia que se relaciona con la primera, que se llama convergencia sigma. Esta concepción de convergencia está estrechamente relacionada con una idea de dispersión del ingreso per cápita entre grupos de países a lo largo del tiempo. Estos dos conceptos fundamentales se pueden resumir de la siguiente manera:

Convergencia Beta: establece la relación inversa entre la tasa de crecimiento y el nivel inicial de ingreso per cápita. Esto implica un crecimiento más rápido para las naciones pobres.

Convergencia Sigma: ocurre cuando la dispersión del ingreso per cápita tiende a disminuir con el tiempo, es decir, este indicador expresa un tipo de inequidad en la distribución del ingreso.

En contraste, dado el progreso realizado por Barro y Sala-i-Martin en el análisis empírico de la convergencia entre países, Quah (1993) argumenta que no es necesario hablar sobre un punto específico donde convergen las economías, pero en estas regiones se forman, a largo plazo, grupos de naciones ricas y pobres. Por lo tanto, propone una nueva metodología que consiste en la elaboración de una matriz de movilidad mediante el uso del ingreso per cápita para establecer una convergencia hacia dos niveles de ingreso, en lugar de un solo estado. Este trabajo en el campo de la dinámica a largo plazo ha proporcionado una contribución importante a la comprensión de las tendencias seguidas por un mundo dividido entre ricos y pobres, y actualmente, este enfoque continúa siendo utilizado en el análisis de convergencia.

En el contexto mundial, el estudio realizado por Miller y Upadhyay (2002) para una muestra conjunta de países desarrollados y en desarrollo, encuentra evidencia estadística que respalda la convergencia $\beta$ absoluta y condicional de la productividad total de los factores, pero solo la convergencia condicional del PIB real por trabajador. Además, Desli y Gkoulgkoutsika (2019) estudian las economías de mayores ingresos del mundo según la clasificación del Banco Mundial, durante el período 1980-2016 y concluyen que el grupo de las economías de mayores ingresos del mundo participa efectivamente en un proceso de convergencia en curso, aunque la crisis financiera podría haberlo perturbado.

Del mismo modo, Chapsa et al. (2015) en su análisis de la convergencia $\beta$ condicional de los ingresos dentro de la UE-15 durante el período 1995 a 2013, incorpora dos variables institucionales, la corrupción y la burocracia. El estudio encuentra evidencia sobre el impacto negativo de la corrupción en el crecimiento y el efecto nulo que tiene la burocracia en el desempeño de los miembros más ricos de la UE. Sin embargo, los países analizados parecen estar en camino de convergencia una vez que el modelo econométrico está controlado por factores económicos como la inversión en capital físico y humano, inflación, consumo público y apertura.

Por otro lado, en América Latina, Azzoni (2001) analiza la evolución de la desigualdad regional en Brasil durante un período de 57 años a través del análisis de convergencia calculado de dos maneras, el modelo neoclásico y el coeficiente de variación. Sus hallazgos indican la presencia de convergencia regional de ingresos 
en Brasil, pero con fluctuaciones significativas en la evolución de la desigualdad en el tiempo. Silva (2010) en su análisis de la convergencia de crecimiento entre los departamentos colombianos durante el período de 1975 a 2000, encuentra evidencia estadística que respalda la hipótesis de convergencia, pero a baja velocidad, alrededor del uno por ciento por año.

Mora (2003) utiliza el enfoque Quah para discutir la idea de los clubes de convergencia y argumenta que, aunque la discusión sobre los resultados del tipo de convergencia sigma y beta es razonable, la forma de cálculo de M (núcleo estocástico) es muy discutible, por lo tanto, el análisis de transición entre países es limitado porque se reduce a una cadena de Markov de un solo paso. Además, Islam (2003) destaca el beneficio de utilizar el enfoque de Quah para analizar la convergencia en estratos, sin embargo, el autor advierte que el análisis debe complementarse con otras herramientas estadísticas para proporcionar una descripción más adecuada de las tendencias en convergencia y transición. El uso de cadenas de Markov se ha extendido y en algunos casos mejorado por el uso de diferentes variables, Moncada e Hincapié (2013) usaron cadenas de Markov para la construcción de matrices de probabilidades de transición de Markov clásicas y espaciales, con lo que los autores concluyen que existe convergencia en la calidad de vida medida por el indicador de la calidad de vida de las comunas y los municipios de Medellín para el período 2004-2011.

En el presente estudio, estos tres enfoques se abordan en el siguiente orden: matriz de movilidad de Quah, convergencia $\sigma$ y, posteriormente, convergencia $\beta$, para lograr los objetivos de la investigación.

\section{Materiales y métodos}

Con respecto a los datos, el presente trabajo empírico utiliza datos del Valor Agregado Bruto (VAB) para 220 de 221 cantones que conforman Ecuador, ya que no es posible incluir Quinsaloma porque fue creado en 2007 y no hay datos para tres períodos consecutivos. La sección temporal considerada incluye el período 2007 a 2017 en virtud de la disponibilidad de datos. La información es proporcionada por las estadísticas oficiales del Banco Central del Ecuador (BCE), que es en términos nominales, siendo necesaria su transformación a términos reales, por lo tanto, de los deflactores implícitos del VAB por industria, procedí a calcular los valores reales de la serie en miles de dólares estadounidenses a precios de 2007. Además, la población anual estimada, requerida para expresar las variables en términos per cápita, se obtuvo del Instituto Nacional de Estadística y Censos de Ecuador (INEC).

Por otro lado, para realizar la estimación econométrica del modelo de convergencia beta condicional, se debe recopilar información sobre las características de cada cantón que ayudan a explicar las disparidades territoriales. Para ello, se utilizan cuatro variables obtenidas de las estadísticas oficiales publicadas por el INEC en su base de datos del Censo de Población y Vivienda 2010, y por la Corporación de Electricidad del Ecuador (CONELEC) para el año 2012. Primero, el porcentaje de viviendas en condiciones de habitabilidad aceptable se define como el número de viviendas cuyas condiciones de vida se consideran aceptables a partir de la combinación de los materiales predominantes de piso, pared y techo; y, el estado de esos 
materiales, expresado como un porcentaje del total de viviendas para un cantón. En segundo lugar, la tasa de analfabetismo, definida como el porcentaje de la población de 15 años o más que no puede leer, escribir o comprender un texto simple y breve sobre su vida diaria, en el período de la encuesta. Tercero, la escolaridad promedio de la población de 24 años o más se define como el número promedio de años aprobados en instituciones de educación formal, para personas de 24 años o más en un territorio específico. Finalmente, la Energía facturada de las residencias se expresa en gigavatios hora $(\mathrm{Gwh})$ para un territorio específico. Cabe señalar que esta última variable se trabaja como un logaritmo para facilitar su interpretación.

\section{Matriz de movilidad de Quah}

El presente trabajo sigue la misma metodología propuesta por Quah (1993). En su trabajo, el autor transforma el ingreso per cápita de los países analizados en fracciones del ingreso per cápita promedio mundial y establece 5 categorías que se clasifican de la siguiente manera:

- Categoría 1/4: corresponde a economías cuyo nivel de ingreso per cápita es menor o igual a $1 / 4$ del promedio mundial

- Categoría 1/2: esta categoría cubre aquellas economías que tienen un ingreso per cápita mayor que $1 / 4$ del promedio mundial y menor o igual a $1 / 2$ del promedio mundial.

- Categoría 1: todas las economías cuyo ingreso per cápita es mayor a la 1/2 del promedio mundial y menor o igual al valor del promedio mundial se encuentran aquí.

- Categoría 2: esta categoría significa que el ingreso per cápita de la economía es más alto que el promedio mundial y menor o igual a 2 veces el mismo promedio global.

- Categoría $\infty$ : aquí se ubican las economías cuyo nivel de ingreso per cápita es superior a 2 .

Una vez que se ha formado la matriz resultante, los valores que están fuera de la diagonal principal muestran el cambio de estado de las economías, con respecto a su situación inicial.

\section{Convergencia Sigma}

Según Barro y Sala-i-Martin (2004), la convergencia sigma se puede expresar como:

$$
\sigma^{2}=\left(\frac{1}{N}\right) \sum\left[\log \left(y_{i, t}\right)-\mu_{t}\right]^{2}
$$

Donde $y_{i, t}$ denota el VAB per cápita de la economía i en el año t y $\mu_{t}$ es la media muestral de $\log \left(y_{i, t}\right)$. Sin embargo, si N es lo suficientemente grande, entonces la varianza de la población se aproxima a la muestra. Dado que este estudio considera todos los cantones en Ecuador, la fórmula a utilizar es la ecuación número 1 con la varianza en población. 


\section{Convergencia beta condicional}

Para probar la hipótesis de convergencia en términos per cápita, a la misma tasa de crecimiento del ingreso y al mismo nivel de capital (estado estacionario), y si las disparidades económicas iniciales tienden a desaparecer (conocido como "convergencia $\beta$ absoluta” o incondicional) Sala-i-Martin (1994), propone el siguiente modelo econométrico:

$(1 / T)^{*} \log \left(y_{i T} / y_{i 0}\right)=\alpha-\left[\frac{1-e^{-b T}}{T}\right] * \log \left(y_{i 0}\right)+w_{i 0, T}$

Sin embargo, dado que en un grupo de economías $\mathrm{i}=1.2 \ldots \mathrm{N}$; la tasa de crecimiento del ingreso per cápita entre el año t y t-1 se puede expresar como $g_{i t}=\log \left(y_{i, t}\right)-\log \left(y_{i, t-1}\right)$, y la hipótesis de convergencia en este enfoque expresa que la tasa de crecimiento es una función negativa del nivel de ingresos en el período inicial, la ecuación (2) puede reexpresarse como:

$g_{i t}=\alpha-\beta \log \left(y_{i 0}\right)+\mu_{i t}$

La ecuación (3) es utilizada ampliamente por la literatura económica en estudios empíricos (Barro y Sala-i-Martin, 2004). Esta ecuación implica que la tasa de crecimiento promedio de la producción per cápita del territorio i en el período comprendido entre 0 y $\mathrm{T} g_{i t}$ tiene una relación negativa con el nivel de producción per cápita en el período inicial $y_{i 0}$. Donde $\alpha$ es una constante, $\beta$ es la velocidad de convergencia y $\mu$ el error de estimación.

Por otro lado, para contrastar la convergencia condicional o relativa, un conjunto de variables $\mathrm{P} X_{i 0}$ puede ser agregado a la ecuación (3), que teóricamente afectan el estado estacionario de cada territorio, lo que resulta en:

$g_{i t}=\alpha-\beta \log \left(y_{i 0}\right)+\sum_{p=1}^{P} \varphi_{p} X_{i 0}+\mu_{i t}$

Tanto la ecuación (3) como la ecuación (4) se estimarán mediante el método de mínimos cuadrados ordinarios.

\section{Resultados de la investigación y discusión}

Esta sección presenta una descripción analítica de la información que se utilizará, así como los resultados obtenidos de la aplicación de los enfoques propuestos junto con un análisis de las implicaciones en términos de crecimiento y desarrollo económico.

\subsection{Análisis de los datos}

Históricamente, las áreas más favorecidas con la implementación de políticas públicas han sido aquellas donde se concentra una gran parte de la actividad económica, ya sea porque es una ciudad capital (como en el caso de Quito) o porque tiene puertos marítimos a través de los cuales ingresa la mercadería (como en el caso de Manta, Guayaquil y Bolívar) o debido al hecho de que fueron lugares de los primeros asentamientos coloniales (como es el caso de Cuenca) o porque son lugares de desbordante 
actividad turística (como en el caso de Galápagos). Esta polarización hace que estos lugares se conviertan en puntos de destino de grandes flujos migratorios o movimientos de la fuerza laboral, ya que la actividad dinámica generada en estos sitios es alta y exige una gran cantidad de recursos. No obstante, a pesar de las medidas adoptadas por los gobiernos de turno, que apuntan a reducir la brecha entre las áreas deprimidas y desarrolladas (como el bono de desarrollo humano y las becas escolares), estas políticas a menudo han estado sujetas a continuas variaciones. Todo esto ha contribuido a hacer que estas polarizaciones sean aún más drásticas con el tiempo. Por lo tanto, para evaluar el desempeño de la economía en general en términos de convergencias, es importante analizar las tendencias que han tenido las principales variables macroeconómicas del Ecuador durante el período de análisis considerado en este estudio. de los precios de las materias primas que exporta Ecuador (como el petróleo) y el populismo de las principales autoridades.

Figura 1. Principales variables macroeconómicas per cápita (dólares estadounidenses 2007)

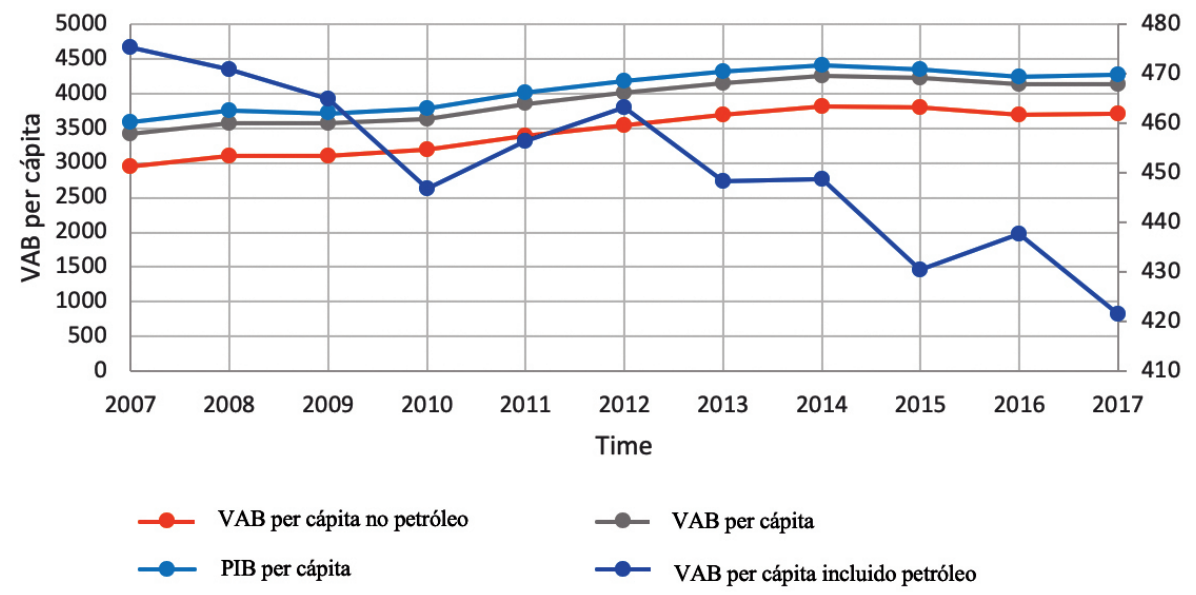

Fuente: BCE, 2019; INEC, 2019.

La figura 1 muestra una alta fluctuación en el valor agregado bruto (VAB) debido a la producción de petróleo. Ecuador se ha caracterizado por ser un país altamente dependiente del petróleo y después del auge del petróleo después de 2007 —período en el que el precio de venta por barril de petróleo alcanzó alrededor de USD \$ 121.66 (BCE, 2009, p. 1) el valor agregado de esta industria ha estado disminuyendo drásticamente. Además, se puede ver que el VAB total sigue una tendencia muy similar al PIB per cápita. Por lo tanto, el Valor Agregado Bruto Total se utilizará en el estudio empírico dado que no hay datos del PIB a nivel cantonal.

Por otro lado, también es importante analizar la situación de los cantones en términos de VAB y su relación con el crecimiento experimentado durante el período analizado. Cabe señalar que este trabajo se realiza con 220 cantones, ya que no es 
posible incluir el cantón Quinsaloma en la provincia de Los Ríos porque se creó en 2007 y no hay datos para tres períodos consecutivos.

Figura 2. Tasa de crecimiento anual 2007-2017

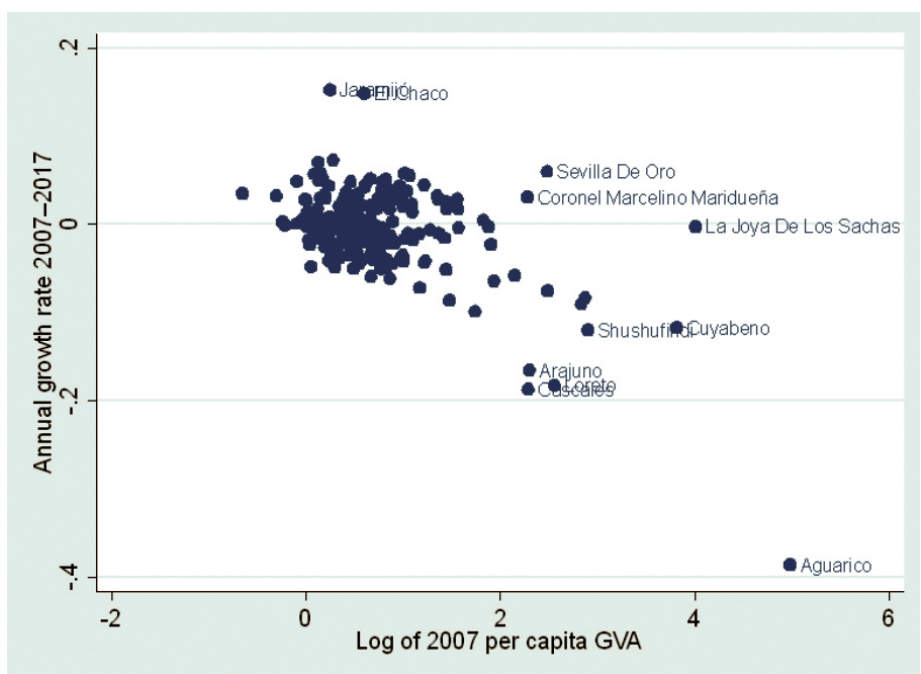

Fuente: BCE, 2019; INEC, 2019.

Como se puede ver en la Figura 2, existe la presencia de ciertos datos que podrían considerarse atípicos, por lo tanto, se debe analizar la situación de 6 cantones en particular: Jaramijó, El Chaco, La Joya de los Sachas y Aguarico. De estos, solo dos pertenecen a la misma provincia, por lo que se puede descartar una posible influencia geográfica.

Figura 3. Tendencias en los cantones señalados (Miles de dólares USA 2007)

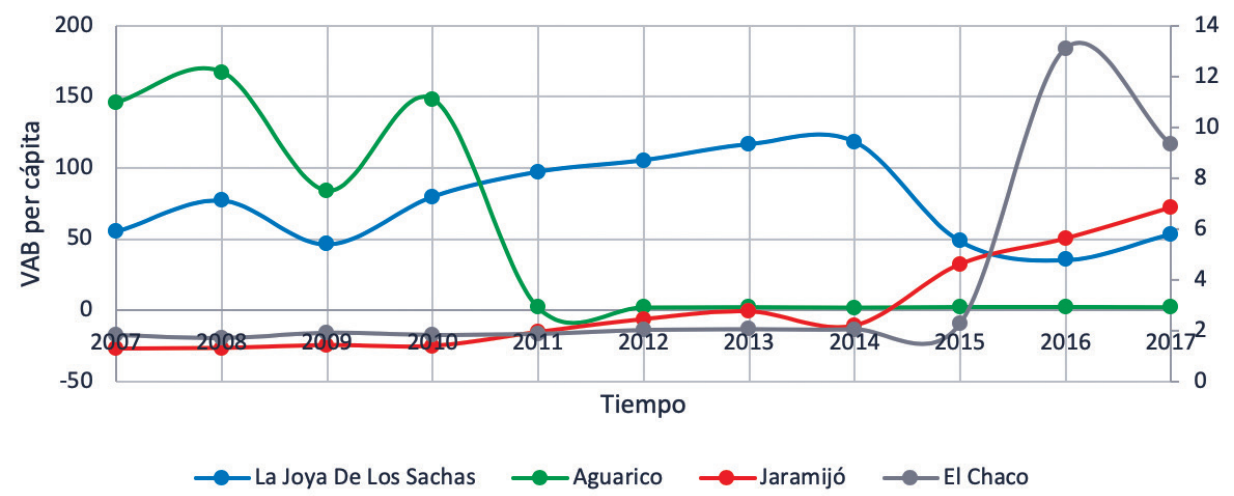

Fuente: BCE, 2019; INEC, 2019. 
Los datos más extremos, en sentido negativo, que se muestran en la Figura 2 pertenecen al cantón Aguarico. La Figura 3 muestra que este cantón registró un alto VAB que ha ido disminuyendo significativamente con el tiempo. Sin embargo, según fuentes oficiales y la prensa local, este cantón no ha experimentado estragos debido a fenómenos naturales, choques externos, migración o disturbios de cualquier otro tipo durante el período de análisis. Se encuentra en la región amazónica de Ecuador, y debe tenerse en cuenta que históricamente las ciudades y comunidades de esa región se han caracterizado por ser distantes y perennemente olvidadas por los gobiernos centrales. Esto produce una limitación severa para el desarrollo; sin embargo, estos datos no pueden clasificarse como atípicos, ya que no existe un factor exógeno que pueda haberlos distorsionado.

Los datos encontrados en el extremo superior de la Figura 2 corresponden a Jaramijó y El Chaco, que se encuentran en las provincias de Manabí y Napo, respectivamente. La Figura 3 muestra que el primero de ellos muestra un patrón de crecimiento sostenido que se vuelve más pronunciado a partir de 2014. Aunque Manabí fue golpeado fuertemente en 2016 por un terremoto, el VAB de este cantón no exhibe una fuerte recesión. Por el contrario, El Chaco, perteneciente a la región amazónica de Ecuador, se ha beneficiado de los programas de construcción de carreteras y autopistas, y, según información de la prensa local, debido a las iniciativas del gobierno actual en ese momento la actividad turística también fue estimulada. Sin embargo, no se puede asegurar que estas iniciativas hayan tenido un impacto que podría generar un crecimiento económico sostenido a largo plazo, ya que el cambio en la tendencia que se muestra en la Figura 3 solo es evidente durante la última parte del período de análisis. No se encuentra evidencia de que estos dos cantones sean atípicos.

Por otro lado, el caso de La Joya de los Sachas, un cantón de la provincia de Orellana ubicada en la Amazonía, mantuvo un crecimiento significativo hasta 2014, pero luego muestra una disminución en su patrón. Este caso particular es un área afectada por la actividad minera en 2018, sin embargo, debido a la disponibilidad de datos hasta 2017, no es posible identificar la tendencia de la serie después de este período. Como se explicó anteriormente, este es el caso de varios otros cantones ubicados en el Amazonas. Sin embargo, los datos registrados para este cantón son altos debido a la producción de cacao y mango con nuevas técnicas agrícolas que han permitido exportar estos productos al extranjero (Alvarado, 2015). Además, durante el siglo pasado, las misiones de órdenes de sacerdotes católicos contribuyeron al desarrollo del cantón y, gracias a su potencial turístico, se ha convertido en el destino de una gran cantidad de pensionistas estadounidenses. Debido a este hecho, se puede inferir que la situación que se muestra en la Figura 3 está cerca de la realidad, por lo tanto, no representa un dato atípico y se incluye en este estudio empírico.

En resumen, se rechaza la presencia de datos atípicos que se muestran en la Figura 2, con la excepción del caso de Quinsaloma, cuyas observaciones no pueden incluirse en el análisis, dadas las limitaciones detalladas anteriormente.

\section{Matriz de movilidad de Quah}

Basado en el proceso descrito en la sección metodológica, se desarrolló la matriz de movilidad para los 220 cantones analizados, teniendo en cuenta el valor agregado 
bruto total a precios constantes de 2007. El valor promedio de VAB per cápita entre los cantones alcanzado en 2007 fue de US \$ 3621.00, mientras que en el año 2017 registró un valor de US \$2662.09. Esta premisa parecería mostrar un deterioro general en la economía ecuatoriana, sin embargo, este comportamiento se debe a los altos valores registrados por ciertos cantones en el período inicial, para los cuales la desviación estándar entre los cantones en 2007 es de US \$ 10941.89, mientras que para el año 2017 es menor, con un valor de US \$4106.75. Por esta razón, se rechaza la posibilidad de una inconsistencia en los datos.

Cada celda de la matriz (i, j) debe interpretarse como la probabilidad de que una economía en el estado inicial i transite o se mueva al estado final j. Por lo tanto, los datos en la diagonal principal de la Tabla 1 muestran la probabilidad de permanecer en la misma posición relativa al final del período de análisis; la parte superior muestra una mejora en la situación observable; abajo, se entiende que el rendimiento ha disminuido en el horizonte temporal. La columna final de la tabla 1 brinda información sobre el número total de cantones ubicados en cada categoría en 2007.

Tabla 1. Matriz de movilidad para Ecuador, 2007-2017

\begin{tabular}{|l|l|l|l|l|l|l|}
\hline \multirow{2}{*}{$\begin{array}{c}\text { Posición } \\
\text { en 2007 }\end{array}$} & $\begin{array}{c}\text { Categoría } \\
\mathbf{1 / 4}\end{array}$ & $\begin{array}{c}\text { Categoría } \\
\mathbf{1 / 2}\end{array}$ & $\begin{array}{c}\text { Categoría } \\
\mathbf{1}\end{array}$ & $\begin{array}{c}\text { Categoría } \\
\mathbf{2}\end{array}$ & $\begin{array}{c}\text { Categoría } \\
\boldsymbol{\infty}\end{array}$ & \multirow{2}{*}{ Número } \\
\hline Categoría $1 / 4$ & 0.00 & 1.00 & 0.00 & 0.00 & 0.00 & 4 \\
\hline Categoría 1/2 & 0.01 & 0.48 & 0.49 & 0.02 & 0.01 & 105 \\
\hline Categoría 1 & 0.00 & 0.06 & 0.59 & 0.32 & 0.02 & 81 \\
\hline Categoría 2 & 0.00 & 0.00 & 0.18 & 0.41 & 0.41 & 17 \\
\hline Categoría $\infty$ & 0.00 & 0.08 & 0.23 & 0.23 & 0.46 & 13 \\
\hline Observaciones Totales & & & & & 220 \\
\hline
\end{tabular}

A partir de la primera fila de la Tabla 1, los resultados muestran una probabilidad del $100 \%$ de pasar a un estrato más alto, a un nivel superior a la cuarta parte del promedio nacional de VAB registrado en 2017 pero inferior a la mitad de ese valor. Este resultado muestra que el grupo de cantones más pobres del país ha mejorado su situación durante el período de análisis. En esta categoría se encuentran los cantones 24 de Mayo, Jama (ubicado en Manabí), Huamboya y Taisha (ubicado en Morona Santiago), sin embargo, este último grupo ubicado en la región amazónica de Ecuador ha sido controversial en los temas relacionados con la extracción de petróleo y contaminación del medio ambiente. Como resultado, no es posible garantizar con total certeza que el nivel de crecimiento económico y la calidad de vida de sus habitantes sea mejor hoy, como sugiere la información.

Del mismo modo, la segunda fila de la Tabla 1 muestra que solo el $1 \%$ de los cantones han deteriorado su situación. Sin embargo, el 49\% de todos los cantones ha alcanzado un estrato más alto, y el $2 \%$ ha alcanzado dos categorías superiores, clasificándose así entre el promedio nacional y el doble. Además, esta categoría con- 
tiene el mayor número de observaciones y el mayor porcentaje de transición positiva registrada durante todo el período de análisis.

Los cantones que registraron un valor inferior al promedio nacional, pero superior a la mitad, muestran la mayor probabilidad de permanecer en el mismo estado a nivel general. Sin embargo, el porcentaje de cantones que se movieron a un nivel superior es del 32\%, que es significativamente mayor que el porcentaje de movilidad descendente.

En consecuencia, los cantones cuyo VAB per cápita era más alto que el promedio nacional al comienzo del período, muestran una probabilidad del $41 \%$ de pasar a un estrato superior, es decir, superior al doble del promedio nacional en la posición final. Este valor coincide con la probabilidad de permanecer en el mismo estado. Por otro lado, la probabilidad de pasar a una categoría inferior registra un valor del 18\%. Por lo tanto, se puede decir que los cantones ubicados en esta categoría han mostrado un buen desempeño porque han experimentado movilidad ascendente a un nivel que supera el doble del promedio nacional registrado en ese año.

La categoría que merece especial atención (así como las economías más pobres) es la que incluye a los cantones más ricos. En este caso, los resultados de la tabla 1 indican que ha habido un deterioro significativo de dicho grupo, ya que la probabilidad de dirigirse hacia los dos estados recesivos es del 23\%, y aún más, es evidente que la probabilidad de un descenso de tres estados asciende al $8 \%$. Es importante señalar que esta categoría excluye los cantones que por tradición han sido los que han concentrado la riqueza generada del país, con la excepción de uno (San Cristóbal, Galápagos) debido a sus actividades turísticas (Vásquez, 2015). En consecuencia, la evidencia sugiere que este conjunto de cantones ha deteriorado su nivel de actividad económica.

Los resultados generales muestran que Ecuador ha experimentado una mejora notable durante todo el período de análisis utilizando el enfoque propuesto por Quah. Por otro lado, dado que no hay datos disponibles a nivel cantonal para períodos anteriores y la literatura existente sobre el tema se limita a estudios a nivel provincial para períodos anteriores al auge petrolero experimentado en 2007 y la dolarización en el año 2000, no se puede llegar a una conclusión definitiva sobre el crecimiento económico y la convergencia a nivel regional a largo plazo. No obstante, los resultados de esta investigación concuerdan con los hallazgos presentados en el estudio realizado por Chimbo y Nauta (2012), a nivel provincial para el período 2001-2007, en el que se argumenta que Ecuador exhibe una movilidad positiva, marcando en de esta manera, una continuidad en la tendencia previamente registrada.

\section{Convergencia Sigma}

Siguiendo la metodología plantada por Barro y Sala-i-Martin, la Figura 4 muestra la relación entre la desviación del logaritmo de VAB per cápita registrada a nivel cantonal con respecto al período de tiempo analizado. 
Figura 4. Dispersión de VAB per cápita en Ecuador, 2007-2017

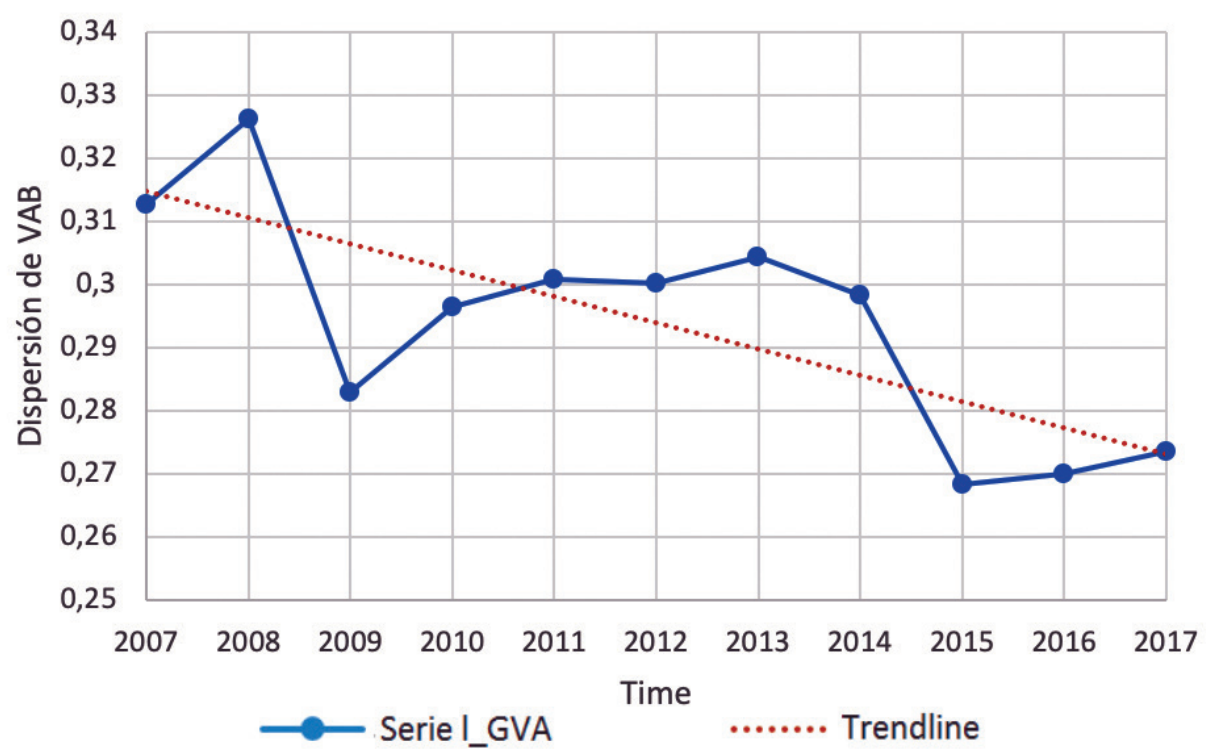

La dispersión registrada al comienzo del período de análisis fue de 0.3126, alcanzando un valor de 0.2735 al final del mismo. Sin embargo, en la Figura 4 se observa que la trayectoria de la serie no ha sido sostenida, mostrando el mayor aumento en 2008 cuyo valor asciende a 0.3262 y, posteriormente, una caída significativa. Además, de 2009 a 2014, la serie muestra un aumento gradual que culmina con otra disminución significativa en 2014 y, posteriormente, durante los últimos tres años exhibe un ligero aumento. A pesar del comportamiento mostrado, la pendiente descendente de la tendencia sugiere que la dispersión ha disminuido con el tiempo. Por lo tanto, los patrones observados de picos son fuertemente compensados por caídas en la dispersión registrada a lo largo del tiempo, evidenciando un claro patrón de convergencia sigma para el caso ecuatoriano en el período analizado.

Este resultado es consistente con los hallazgos presentados en el estudio de Riofrío (2009) a nivel provincial para el período 1993-2007, que argumenta que la tendencia de dispersión registrada a nivel provincial ha disminuido a una tasa que podría considerarse sostenida en el a largo plazo. Como resultado, al considerar estos hallazgos, se puede concluir que Ecuador ha mostrado un desempeño notable en su intento de reducir las disparidades económicas territoriales.

La implicación de este hallazgo sugiere que los esfuerzos realizados por el gobierno de turno para reducir las brechas económicas entre regiones podrían haber tenido el impacto deseado. Sin embargo, es importante tener en cuenta que este período se caracteriza por la estabilidad política en Ecuador, a diferencia de las décadas pasadas, donde ningún presidente pudo completar el período para el que fue elegido, y aún más para ser reelegido en las elecciones generales. Esta estabilidad 
tiene un papel importante en el momento del análisis de convergencia y crecimiento a largo plazo, porque, independientemente de las medidas tomadas, la coherencia y el cumplimiento de un proyecto y plan específicos pueden mostrar resultados positivos a largo plazo, sin ignorar el esfuerzo articulado de administraciones seccionales. Sin embargo, el resultado obtenido no puede atribuirse solo a este factor (estabilidad política), ya que podría haber otras variables y factores presentes para el logro de los resultados expuestos.

\section{Convergencia Beta}

La Tabla 2 muestra los resultados obtenidos de las ecuaciones (3) y (4) utilizando Mínimos Cuadrados Ordinarios. Cabe señalar que los modelos 1 y 2 exhibieron presencia de heterocedasticidad, por lo tanto, las estimaciones se presentan utilizando errores estándar robustos.

Tabla 2. Resultados del modelo de Convergencia absoluta y condicional.

\begin{tabular}{|l|c|c|c|c|}
\hline \multirow{2}{*}{ Variables } & Modelo 1 & Modelo 2 & Modelo 3 & Modelo 4 \\
\cline { 2 - 5 } & Coeficientes & Coeficientes & Coeficientes & Coeficientes \\
\hline$\beta$ & $-0.0362 * * *$ & $-0.0261 * * *$ & $-0.0460 * * *$ & $-0.0467 * * *$ \\
\hline Vivienda & & & $0.0916 * * *$ & $0.0632 *$ \\
\hline Tasa de analfabetismo & & & -0.1047 & \\
\hline lnelec & & & $0.0064 * * *$ & $0.0056 * *$ \\
\hline Promedio de escolaridad & & & & $0.0067 * *$ \\
\hline$\alpha$ & $0.0220 * * *$ & $0.0157 * * *$ & -0.0011 & $-0.0529 * * *$ \\
\hline Observaciones & 220 & 219 & 220 & 220 \\
\hline F-estadístico & 85.597 & 44.5063 & 10.3982 & 11.0893 \\
\hline R-cuadrado & 0.2819 & 0.1702 & 0.4601 & 0.4676 \\
\hline Aic & -770.618 & -804.963 & -827.3727 & -830.4204 \\
\hline Bic & -763.831 & -798.184 & -810.4045 & -813.4523 \\
\hline leyenda: *p<.05; **p $<.01 ; * * * \mathrm{p}<.001$ & & & \\
\hline
\end{tabular}

Primero, es importante resaltar que existe evidencia de convergencia beta absoluta en el nivel cantonal porque el coeficiente obtenido es negativo y estadísticamente significativo, cuyo valor es de alrededor de $3.62 \%$ promedio anual. Además, debido a la presencia de un valor que podría considerarse un valor atípico, perteneciente al cantón Aguarico, el Modelo 2 excluye esta observación para verificar la existencia de posibles cambios en la magnitud de los coeficientes. Los resultados se muestran en la segunda columna de la Tabla 2. La evidencia sugiere que la tasa de convergencia absoluta disminuye a $2.61 \%$, sin embargo, los resultados mantienen su validez y significación estadística. Como resultado, se corrobora la existencia de una convergencia absoluta a nivel cantonal en Ecuador. 
Al comparar los resultados obtenidos con un estudio previo realizado para el período 2007 a 2012 por Mendieta (2015), que encuentra una velocidad de convergencia de alrededor del 1,37\%, se puede ver que el valor informado en este trabajo es mayor. Esta diferencia puede atribuirse al factor tiempo. El presente trabajo considera un período de tiempo más largo, y debido a que este tipo de análisis requiere intervalos de tiempo extensos para mostrar resultados estadísticamente más sólidos, es admisible considerar que la velocidad de convergencia obtenida en esta investigación está más cerca de la realidad.

Por otro lado, es necesario analizar qué variables afectan este proceso de convergencia beta. Para esto, se tiene en cuenta el enfoque de convergencia beta condicional detallado en la sección de metodología y sus resultados se presentan en los Modelos 3 y 4 de la Tabla 2. En el presente trabajo se utiliza un conjunto de características relacionadas con el Infraestructura, educación y consumo de electricidad, con el objetivo de explicar la convergencia condicional de cada territorio. Las variables consideradas para este propósito son: Porcentaje de viviendas en condiciones de habitabilidad aceptable (vivienda), tasa de analfabetismo (tasa de analfabetismo), escolaridad promedio de la población de 24 años y mayor (escolaridad promedio), logaritmo de energía facturada de residencias (lnelec), que se obtienen del Censo de Población y Vivienda 2010, excepto el último, cuyos datos están registrados por CONELEC para 2012. La diferencia entre los modelos mencionados anteriormente radica en la introducción de la variable que se refiere al nivel educativo de la población para los cantones de análisis. En este sentido, la introducción de las variables tasa de analfabetismo y escolaridad promedio en el mismo modelo crearía inconsistencias estadísticas y teóricas. Por esta razón, el Modelo 3 considera la tasa de analfabetismo, mientras que el Modelo 4 considera la escolaridad promedio de la población.

Los resultados que se muestran en la Tabla 2 indican que la velocidad de convergencia condicional es mayor en el caso del Modelo 3, en comparación con el resultado de convergencia absoluta. El coeficiente obtenido es negativo y estadísticamente significativo con un valor de $4.60 \%$ promedio anual a nivel cantonal. Además, las variables de acondicionamiento facturan energía y porcentaje de hogares en condiciones de vida aceptables, tienen coeficientes positivos y estadísticamente significativos, lo que mantiene una congruencia lógica ya que un cantón que demanda un alto consumo de energía eléctrica y tiene una infraestructura física adecuada experimenta una tasa de crecimiento promedio más alta de producción per cápita. Sin embargo, aunque la tasa de analfabetismo no es estadísticamente significativa, su signo parece ser lógico, ya que se espera que una tasa de analfabetismo más alta afecte negativamente la tasa de crecimiento promedio en el período de análisis. Finalmente, una descripción general del modelo permite mostrar que, de acuerdo con el valor del estadístico $\mathrm{F}$, las variables son estadísticamente significativas desde un nivel de significancia del 1\%, además, su inclusión mejora el coeficiente R Cuadrado, bondad de ajuste.

De acuerdo con los resultados de la columna Modelo 4 de la Tabla 2, hay un ligero aumento en la velocidad de convergencia que registra un valor de $4.67 \%$. La estimación econométrica muestra un mejor grado de ajuste y, como en el caso del modelo analizado previamente, la significancia estadística de las variables se mantiene conjuntamente. Sin embargo, a diferencia del modelo anterior, todas las variables 
introducidas en el modelo demuestran ser estadísticamente significativas a nivel individual con un nivel de confianza del $90 \%$. Un análisis más profundo muestra que un aumento en el porcentaje de hogares con características de habitabilidad adecuadas se asocia con un aumento de 0.06 puntos porcentuales en la tasa de crecimiento promedio de producción per cápita y, de la misma manera, un aumento del $1 \%$ en la cantidad facturada de energía eléctrica, produce un aumento promedio estimado de $0.0056 \%$ en la tasa de crecimiento promedio per cápita. Esto corrobora la hipótesis de que, al mejorar las condiciones de la infraestructura doméstica y una mayor demanda de energía eléctrica, tiene el efecto de reducir las asimetrías económicas territoriales en Ecuador. Por otro lado, la educación es un factor clave en el crecimiento y desarrollo económico de los territorios. El coeficiente asociado a esta variable muestra que, cada año adicional de educación, se correlaciona positivamente con un aumento de $0.0067 \%$ en la tasa de crecimiento promedio de la producción de cada territorio. Estas magnitudes son coherentes con el mayor nivel de convergencia, dado que el impacto positivo que producen juntos en el modelo de análisis es positivo, lo que denota su relevancia para el proceso de convergencia.

Un análisis comparativo de los modelos en términos estadísticos permite establecer que, dado el ajuste para la adición de nuevas variables explicativas, la mejor estimación econométrica se presenta en el Modelo 4 de la tabla 2, de acuerdo con Akaike (1974) y Schwarz (1978) Criterios de información bayesianos.

Este conjunto de resultados implica que el gobierno debería, en la medida de lo posible, mejorar las condiciones de vida de la población en términos de infraestructura física y acceso a servicios básicos, así como el nivel educativo de la población. Sin embargo, con el propósito de reducir las brechas económicas entre las diferentes regiones del país, debe tenerse en cuenta que estas variables son solo una muestra de las posibles áreas de intervención y no se descarta la existencia de otros factores no considerados en este estudio que contribuyen a la reducción de las disparidades territoriales. En este sentido, múltiples investigaciones y evidencia empírica señalan la importancia de la educación, así como la inversión en infraestructura, en el crecimiento económico de un país, y aún más en el grado de desigualdad económica y pobreza en un territorio. Estos resultados son consistentes con los hallazgos de Duffy-Deno y Eberts (1991) que muestran la importancia de la inversión en infraestructura y su impacto en el desarrollo regional. Démurger (2001) cuya contribución explica el impacto que el Desarrollo de Infraestructura ha tenido en la reducción de las disparidades regionales en China, y Adshead et al. (2019), que encuentra evidencia de que la planificación de infraestructura a largo plazo es eficiente para cumplir con los Objetivos de Desarrollo Sostenible en Curazao. Además, el estudio realizado por Goetz y Hu (1996) destaca la importancia de la educación como factor clave en la formación de capital humano y su contribución a la velocidad de la convergencia de ingresos. Del mismo modo, Kruss et al. (2015) y Rivza et al. (2015) argumentan que reforzar el proceso educativo y los programas de estudio conduciría a una mejora en las tendencias de crecimiento regional.

Según los resultados, es posible analizar dos aspectos relevantes que contribuyen a explicar el grado de convergencia territorial y su impacto a largo plazo. Primero, Ecuador actualmente muestra grandes deficiencias en términos de infraes- 
tructura física entre cantones, siendo los más desfavorecidos aquellos con un gran porcentaje de población indígena, montubia, afrodescendiente y, principalmente, la región amazónica. Si bien es cierto que durante el período de análisis se ha mantenido la estabilidad política y la inversión en dimensiones sociales como el trabajo, la salud y la educación ha sido visible, aún existen grandes asimetrías entre los territorios que concentran la riqueza del país debido a sus características históricas y geográficas, y aquellos que no tienen tales ventajas. Esto se refleja en los resultados, específicamente en el valor relativamente bajo de la velocidad de convergencia.

En segundo lugar, una medida efectiva e inmediata que se sugiere ampliamente en la literatura económica consiste en un estímulo académico que, como se indicó anteriormente, tiene grandes beneficios tanto a corto como a largo plazo. Sin embargo, en Ecuador, el acceso a la educación formal está fuertemente condicionado en muchos casos al nivel socioeconómico del hogar. Aunque la educación en los niveles primario y secundario es en su mayormente gratuita, su calidad es ampliamente cuestionada. De una forma u otra, existen grandes diferencias entre las escuelas, siendo las mejores ubicadas generalmente en la metrópoli (que concentran la mayor parte de la actividad económica del país), en contraste, aquellas escuelas ubicadas en el sector rural carecen de muchas comodidades. La educación rural es uno de los factores más importantes en el desarrollo regional (Biriescu \& Babaita, 2014), por lo tanto, los gobiernos seccionales y municipales deberían centrar más esfuerzos en fortalecer los programas educativos en el sector rural. Esta realidad produce un incentivo para la migración interna, sin embargo, muchas veces esa decisión está condicionada por la situación económica del hogar (coste de oportunidad) y la percepción de beneficios futuros en relación con el empleo. En este escenario, es muy probable observar que cuando se toma la decisión de migrar, no se hace con el deseo de regresar a la zona de origen ya que, en muchos casos, no hay trabajos que permitan explotar los conocimientos adquiridos. Por otro lado, la decisión de permanecer implica que la continuidad de un bajo nivel educativo en el territorio. Ambos fenómenos contribuyen a que las áreas deprimidas continúen subdesarrolladas mientras que las áreas ricas continúan creciendo, aumentando las asimetrías territoriales dentro del Ecuador.

Por otro lado, un ejercicio matemático, a partir de la magnitud informada del coeficiente beta y el supuesto de crecimiento geométrico utilizado en la hipótesis de convergencia, proporciona una idea sobre el grado de convergencia en términos temporales. Tomando como referencia la velocidad de convergencia absoluta informada por el Modelo 1 de la Tabla 2, es posible determinar que el cantón Salitre de la provincia de Manabí (el más pobre) tomaría aproximadamente 120.86 años para alcanzar la producción promedio per cápita de La Joya de los Sachas de la provincia de Orellana (la más rica). Este resultado muestra que, aunque existe evidencia estadística de convergencia absoluta, esto no es suficiente para cerrar las brechas económicas en Ecuador a corto plazo. Por el contrario, al considerar el coeficiente de convergencia condicional obtenido del Modelo 4 en la Tabla 2, un cantón en términos generales tardaría aproximadamente 14.5 años en promedio para alcanzar el doble de su producción per cápita registrada en el año 2017, ceteris paribus. Estos resultados muestran de manera más clara la magnitud del proceso de convergencia a nivel cantonal en Ecuador y sus implicaciones en términos temporales. Como resultado, 
además de la corroboración empírica de la hipótesis de convergencia beta a nivel cantonal durante el período 2007-2017, se ha demostrado que puede considerarse lenta al analizar su dimensión temporal.

\section{Conclusión}

Los resultados de la presente investigación se pueden agrupar en tres hallazgos principales. En primer lugar, se concluye que Ecuador ha experimentado movilidad interna en un sentido positivo a nivel cantonal, es decir, según la matriz de movilidad de Quah, una parte significativa de los cantones analizados muestra una mejora en su situación final, situándose en categorías superiores a las reportadas inicialmente. Las implicaciones de este resultado sugieren que las políticas aplicadas y la estabilidad política experimentada en este período (dada la coincidencia con el período de gobierno en ese momento) podrían haber contribuido a mejorar la situación económica del país y su nivel de crecimiento económico. Sin embargo, se necesitan más estudios para asegurar la importancia de la variable política.

Segundo, la evolución en el grado de dispersión en la producción per cápita muestra que Ecuador ha experimentado una reducción sistemática en la variabilidad de este indicador a lo largo del tiempo, lo que respalda la existencia de convergencia sigma. En este sentido, la economía ecuatoriana en su conjunto muestra una mejora continua durante el período de análisis.

Tercero, la evidencia empírica corrobora la existencia de convergencia beta absoluta y condicional a nivel cantonal para el período 2007-2017. En el primer caso, la velocidad de convergencia asciende al 3.62\% promedio anual, mientras que, en el segundo caso, al considerar la infraestructura física de los hogares, su consumo de energía eléctrica y su nivel educativo, se obtienen resultados altamente significativos y la velocidad de convergencia alcanza el $4.67 \%$ porcentaje anual. Por lo tanto, se concluye la existencia de convergencia beta para el caso ecuatoriano.

Por otro lado, la combinación de los enfoques utilizados en este estudio permite garantizar que, aunque los principales hallazgos son favorables y muestran un buen desempeño de la economía ecuatoriana, aún no son suficientes para cerrar efectivamente las brechas territoriales en el mediano plazo y probablemente a largo plazo. En términos generales, Ecuador ha mostrado una mejora sistemática en su situación económica a lo largo del tiempo, sin embargo, depende del gobierno de turno emprender nuevas iniciativas más efectivas que garanticen una reducción efectiva de las asimetrías económicas entre los territorios del país, ya que el período analizado se caracteriza por un auge petrolero, por lo tanto, sería importante verificar que el cierre evidente de las brechas en este estudio no se revierta en los próximos años.

Las principales reflexiones para los formuladores de políticas económicas en Ecuador consisten en el énfasis que se debe poner en mejorar la calidad de la infraestructura del hogar y el nivel educativo de las personas como factores relevantes para el desarrollo, entre otros. Sin embargo, no se descarta la incidencia de otras variables que no se incluyeron en este estudio y su relevancia en la reducción de las disparidades económicas en un territorio. Además, la evidencia empírica proporcionada 
por el presente estudio para el caso ecuatoriano durante el período 2007-2017 está respaldada por la literatura económica e investigaciones realizadas sobre este tema.

Las principales limitaciones encontradas fueron en relación con la disponibilidad de datos. Por el momento, Ecuador no mantiene un registro a nivel cantonal durante años fuera del período de análisis considerado en este estudio. Del mismo modo, las fuentes estadísticas oficiales no tienen información a nivel cantonal sobre los factores relevantes que la literatura económica sugiere incluir en un análisis de convergencia condicional. Sin embargo, la información utilizada en esta investigación permitió obtener resultados estadísticamente sólidos.

Finalmente, investigaciones futuras podría encargarse de períodos de tiempo más extensos y analizar la posibilidad de incluir otros factores condicionantes que ayuden a explicar las asimetrías territoriales presentes en Ecuador.

\section{Referencias}

Adshead, D., Thacker, S., Fuldauer, L. I., \& Hall, J. W. (2019). Delivering on the Sustainable Development Goals through long-term infrastructure planning. Global Environmental Change, 59. https://doi.org/10.1016/j.gloenvcha.2019.101975

Akaike, H. (1974). A New Look at the Statistical Model Identification. IEEE Transactions on Automatic Control, 19(6), 716-723. https://doi.org/10.1109/TAC.1974.1100705

Alvarado, R. J. (2015, diciembre). Potencial de las empresas petroleras para el desarrollo local amazónico: análisis a partir de su incidencia en el sector agropecuario del cantón la Joya de los Sachas. (Tesis de maestría). Quito: Repositorio Digital FLACSO Ecuador. Recuperado de: https://bit.ly/2HgXyRM

Azzoni, C. (2001). Economic growth and regional income inequality in Brazil. The Annals of Regional Science, 35, 133-152. https://doi.org/10.1007/s001680000038

Barro, R., \& Sala-i-Martin, X. (1991). Convergence Across States and Regions. Brookings Papers on Economic Activity, 22(1), 107-182.

Barro, R., \& Sala-i-Martin, X. (1992a). Convergence. Journal of Political Economy, 100(2), 223251. https://doi.org/10.1086/261816

Barro, R., \& Sala-I-Martin, X. (1992b). Public Finance in Models of Economic Growth. The Review of Economic Studies, 59(4), 645-661. https://doi.org/10.3386/w3362

Barro, R., \& Sala-i-Martin, X. (2004). Economic Growth (2nd ed.). Cambridge: Massachusetts Institute of Technology.

BCE (diciembre de 2009). Banco Central del Ecuador. Recuperado de: https://bit.ly/38o4QyZ

BCE (mayo de 2019). Banco Central del Ecuador. Recuperado de: https://bit.ly/39sh7T6

Biriescu, S., \& Babaita, C. (2014). Rural Education, an Important Factor of Regional Development in the Context of Local Government Strategies. Procedia-Social and Behavioral Sciences, 124, 77-86. https://doi.org/10.1016/j.sbspro.2014.02.462

Chapsa, X., Tsanana, E., \& Katrakilidis, C. (2015). Growth and Convergence in the EU-15: More Evidence from the Cohesion Countries. Procedia Economics and Finance, 33, 55-63. https://doi.org/10.1016/S2212-5671(15)01693-7

Chimbo, J., \& Nauta, F. (2012). Movilidad de las provincias del Ecuador desde el punto de vista del VAB, 2001-2007. Matriz de Quah. Cuenca: Degree Work, Universidad de Cuenca. Recuperado de: https://bit.ly/2SnA7fQ

Démurger, S. (2001). Infrastructure Development and Economic Growth: An Explanation for Regional Disparities in China? Journal of Comparative Economics, 29(1), 95-117. https:// doi.org/10.1006/jcec.2000.1693 
Desli, E., \& Gkoulgkoutsika, A. (2019). Economic convergence among the world's top-income economies. The Quarterly Review of Economics and Finance. https://doi.org/10.1016/j. qref.2019.03.001

Duffy-Deno, K. T., \& Eberts, R. W. (1991). Public infrastructure and regional economic development: A simultaneous equations approach. Journal of Urban Economics, 30(3), 329-343. https://doi.org/10.1016/0094-1190(91)90053-A

Goetz, S. J., \& Hu, D. (1996). Economic growth and human capital accumulation: Simultaneity and expanded convergence tests. Economics Letters, 51(3), 355-362. https://doi. org/10.1016/0165-1765(96)00827-0

INEC (2019, mayo). Instituto Nacional de Estadísticas y Censos. Recuperado de: https://bit. ly/39CDeGR

Islam, N. (2003). What have We Learnt from the Convergence Debate? Journal of Economic Surveys, 17(3), 309-362. https://doi.org/10.1111/1467-6419.00197

Kruss, G., McGrath, S., Petersen, I.-h., \& Gastrow, M. (2015). Higher education and economic development: The importance of building technological capabilities. International Journal of Educational Development, 43, 22-31. https://doi.org/10.1016/j.ijedudev.2015.04.011

Lucas, R. (1988). On the mechanics of economic development. Journal of Monetary Economics, 22(1), 3-42.

Mendieta, R. (2015). La hipótesis de la convergencia condicional en Ecuador: un análisis a nivel cantonal. Retos. Revista de Ciencias de la Administración y Economía, 5, 13-26. https:// doi.org/10.17163/ret.n9.2015.01

Miller, S. M., \& Upadhyay, M. P. (2002). Total factor productivity and the convergence hypothesis. Journal of Macroeconomics, 24(2), 267-286. https://doi.org/10.1016/S0164-0704(02)00022-8

Moncada, J., \& Hincapié, D. (2013). Convergence of the Quality of Life in Medellin 2004-2011. A Spatial Nonparametric Analysis. Ensayos sobre Política Económica, 30(70), 267-314. doi:https://doi.org/10.1016/S0120-4483(13)70034-4

Mora, J. J. (2003). Crecimiento y convergencia: A propósito de Quah. Estudios Gerenciales, 89, 57-72. Recuperado de: https://bit.ly/2SoMmZW

Quah, D. (1993). Empirical cross-section dynamics in economic growth. European Economic Review, 37(2-3), 426-434. https://doi.org/10.1016/0014-2921(93)90031-5

Rebelo, S. (1991). Long Run Policy Analysis and Long Run Growth. Journal of Political Economy, 99(3), 500-521.

Riofrío, L. (2009). Capital humano y procesos de convergencia en el Ecuador. Degree Work. Loja: Universidad Técnica Particular de Loja. Recuperado de https://bit.ly/39x6zlK

Rivza, B., Bikse, V., \& Brence, I. (2015). Evaluation of Higher Education Study Programmes and their Development Trends as Drivers of Regional Growth. Procedia Economics and Finance, 26, 643-650. https://doi.org/10.1016/S2212-5671(15)00804-7

Romer, P. (1986). Increasing Returns and Long-Run Growth. Journal of Political Economy, 94(5), 1002-1037.

Sala-i-Martin, X. (1994). Cross-sectional regressions and the empirics of economic growth. European Economic Review, 38(3-4), 739-747.

Schwarz, G. (1978). Estimating the Dimension of a Model. The Annals of Statistics, 6(2), 461-464. https://doi.org/10.1214/aos/1176344136

Silva, A. (2010). Economic Growth and Poverty Reduction in Colombia. Frankfurt Am Main: Peter Lang AG. https://doi.org/10.3726/978-3-653-00274-4

Solow, R. (1956). A Contribution to the Theory of Economic Growth. The Quarterly Journal of Economics, 70(1), 65-94.

Vásquez, M. E. (2015). Propuesta para implementar un ecoturismo rural en la isla San Cristóbal. (Tesis de Pregrado). Guayaquil, Ecuador. Recuperado de: https://bit.ly/31QaN5r

Young, A. (1991). Learning by Doing and the Dynamic Effects of International Trade. The Quarterly Journal of Economics, 106(2), 369-405. 